\title{
Effects of Local Vibration on Shoulder Horizontal Adduction and Internal Rotation Range of Motion in Subject with Posterior Shoulder Tightness
}

\author{
Sung-hoon Jung, PT, Ph.D ${ }^{1}$; Sung-min Ha, PT, Ph.D ${ }^{2}$ \\ ${ }^{1}$ Department of Physical Therapy, College of Health Science, Yonsei University, South Korea \\ ${ }^{2}$ Department of Physical Therapy, College of Health Science, Sangji University, South Korea
}

Background In recent years, the use of local vibration (LV) devices is increasing to increase the elongation and reduce stiffness of muscle tissue in the field of rehabilitation and sports. However, to date, no research has been conducted on the therapeutic effect of local vibration therapy devices.

Purpose We investigated the effects of local vibration on the posterior shoulder tightness, specifically, range of motion (ROM) of the shoulder horizontal adduction (HA) and internal rotation (IR).

Study design One group pre-post study

Methods Thirteen subjects with limitation in passive shoulder HA and IR with less than $50 \%$ of normal ROM were recruited from university.

Results All continuous variables were found to approximate a normal distribution (KolmogorovSmirnov Z-test, $p>0.05$ ). The shoulder HA and IR ROM were significantly different between preand post-LV $(p<0.05)$.

Conclusions Based on the results of this study, it was found that it is effective to apply LV to areas with shortening and stiffness of muscle or fascia tissue.

Key words Local vibration; Posterior shoulder tightness; Shoulder horizontal adduction; Shoulder internal rotation.
JMST
2020; 4(2): 66-69
Published Online
Dec 31, 2020
pISSN 2635-8573
eISSN 2635-8581
Article History
Received 4 Oct 2020
Revised 18 Oct 2020
(1st)
Revised 27 Oct 2020
(2nd)
Accepted 28 Oct 2020

\section{CONTACT}
hsm98@sangji.ac.kr Sung-min Ha,
Department of Physical
Therapy, College of
Health Science, Sangji
University, South Korea
This is an Open-Access article distributed under the terms of the Creative Commons Att- ribution Non-Commercial Li- cense (http://creativecommons. org/licenses/by-nc/4.0) which org/licenses/by-nc/4.0) which permits unrestricted non-co- minnercial use, distribution, and reproduction in any me dium, provided the origina work is properly cited.

\section{INTRODUCTION}

Passive static stretch has been widely used for decreasing posterior shoulder muscles (infraspinatus, and posterior deltoid) tightness, which is lead to restricted joint mobility, shoulder impingement, labral lesion, and rotator cuff tear. ${ }^{1-3}$ To restore the shortened or stiff muscle to its normal length or tension, a stretching method is generally used, but there is a limitation in actual movement recovery. ${ }^{4}$ Increased muscle flexibility after stretching is due to sensory modification, rather than increased length of muscle, an idea referred to as theory of sensory. ${ }^{5-6}$ Therefore, alteration in the sensitivity (by sensory receptor) for stretching must be made in the end range of stretching. ${ }^{7-8}$

When a general stretching method is performed, the ex- tensibility of the muscles in the end range is increased due to the reflex action of the sensory receptors for pain and stretching. ${ }^{9}$ Various therapeutic tools have been widely used to solve this problem and improve the therapeutic effect of stretching. ${ }^{10}$ Local cryotherapy reduces the uncomfortable sensation that patients feel when stretching and increases the therapeutic effect. ${ }^{11}$ Instrument assisted soft tissue mobilization (IASTM) using Graston technique is effective for treating soft tissue dysfunction, increased mobility of soft tissue, fibroblast production, and morphological changes in muscle. ${ }^{12}$ In recent years, the use of vibration devices is increasing to increase the elongation and reduce stiffness of muscle tissue, in the field of rehabilitation and sports. Compared to cryotherapy or IASTM, it can be easily applied and relaxes soft tissues in a short time. 
However, to date, no research has been conducted on the therapeutic effect of local vibration therapy devices. Thus, we investigated the effects of local vibration on the posterior shoulder tightness, specifically range of motion (ROM) of the shoulder horizontal adduction (HA) and internal rotation (IR). We hypothesized local vibration would decrease posterior shoulder tightness (ROM of the HA and IR).

\section{METHOD}

\section{Participants}

Thirteen subjects (13 male) with limitation in passive shoulder HA and IR were recruited from university (Those with less than $50 \%$ of normal ROM) (Table 1). Exclusion criteria were (1) history of shoulder surgery, (2) history of shoulder fracture, (3) congenital shoulder abnormalities, and (4) ongoing shoulder pain. Prior to the experiment, all experiment procedure were explained to the subjects. They participated voluntarily and provided written informed consent. Each provided and signed informed consent on a form approved by the Yonsei University, Mirae Campus, Institutional Review Board (approval number: 1041849-201701BM-008-02).

\section{INSTRUMENTATION}

\section{Local vibration device}

The local vibration massage device (Hypervolt, Hyperice USA), which was applied to increase the mobility of the area with posterior shoulder tightness (Figure 1), was used as the most comfortable circumferential attachment for the patient before application. Step 1, the lowest of the three speeds, was applied while moving to the posterior shoulder muscle (posterior deltoid, infraspinatus, and teres minor muscles).

\section{Camera}

A smartphone camera (Galaxy note 9, Samsung, Republic of Korea) was installed on a tripod at a 3-meter distance from each subject and aligned to the shoulder joint, so it

Table 1. General characteristics of subjects

$(n=13)$

\begin{tabular}{cc}
\hline Variable & Mean \pm SD \\
\hline Age (yrs) & $23.9 \pm 1.5$ \\
Height $(\mathrm{cm})$ & $168.8 \pm 7.0$ \\
Weight $(\mathrm{kg})$ & $66.6 \pm 14.1$ \\
\hline
\end{tabular}

$\mathrm{SD}=$ standard deviation

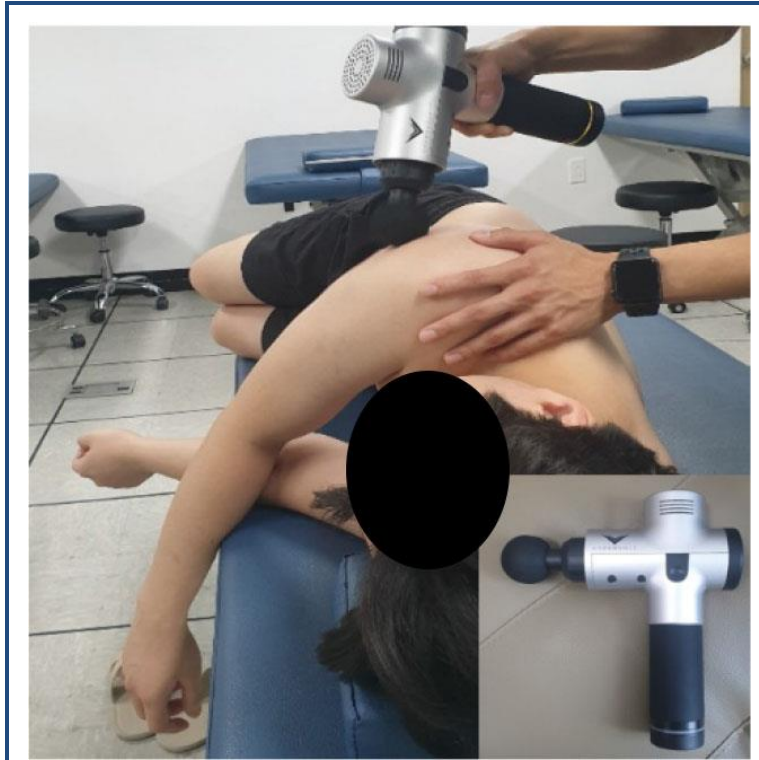

Figure 1. Application of local vibration device.

was only possible to record the picture when the smartphone camera was perpendicular to the ground. The images for photographic measurement were captured at the end-range of shoulder HA and IR. The captured image was processed using the Image J program (version 1.43, National Institutes of Health, Bethesda, Maryland) which can measure the joint angle, in degrees, between two lines. The HA ROM (angle) was formed by joining the acromion process, lateral epicondyle of humerus, and a horizontal line. And, the IR ROM (angle) was formed by joining the ulnar styloid process, the olecranon process of the ulna, and a vertical line that passed through the olecranon process of the ulna.

\section{Procedure}

Subject lie down on the table with the top undressed and the top naked. The examiner measured initial ROM of the subject's shoulder HA and IR. Measurement was made with the humerus head fixed to prevent compensation movement that may occur during measurement. After measuring the initial ROM, a local vibration device was used to treat the posterior shoulder muscle (posterior deltoid, infraspinatus, and teres minor muscles) with local vibration for 5 minutes (Figure 1). After local vibration therapy, the subject's shoulder horizontal adduction and internal rotation ROM were measured to confirm the therapeutic effect.

\section{Statistical analysis}

The statistical analysis were performed using Statistical Software package for the Social Science (SPSS) version 23.0 (SPSS Inc., Chicago, IL), and the level of statistical 
significance was set at $p<0.05$. To confirm a normal distribution, the Kolmogorov-Smirnov test was used. To investigate the effect of effects of LV on the posterior shoulder tightness (ROM of the HA and IR), a paired $t$-test was used.

\section{RESULT}

All continuous variables were found to approximate a normal distribution (Kolmogorov-Smirnov Z-test, $p>0.05$ ). The shoulder HA and IR ROM were significantly different between pre- and post-LV $(p<0.05$, Table 2$)$.

\section{DISCUSSION}

The use of LV therapy devices has been recently used to improve musculoskeletal rehabilitation and athlete performance. Although LC is widely used in the clinical field, it is difficult to find studies dealing with its therapeutic effect. The purpose of this study was to compare shoulder HA and IR between pre- and post-LC. The results of this study showed that the shoulder HA and IR ROM were significantly different between pre- and post-LV $(p<0.05)$.

There are some explanation for the results. According to previous studies, high frequency vibration stimulation stimulates type 1a sensory afferent (dynamic muscle spindle), but reduces type $1 \mathrm{~b}$ sensory afferent (golgi tendon organ) and type 2 afferent (static muscle spindle afferent) stimulation. ${ }^{13}$ At the same time, LV exhibits inhibition of agonist muscle, tonic contraction (known as the tonic vibration reflex), and reciprocal inhibition of the antagonist muscle. In addition, LV significantly reduces H-reflex sensitivity. ${ }^{14}$ In particular, pacinian sensory receptors (mechanoreceptor) respond to local vibrations and have a great influence on movement control and muscle activity. ${ }^{15}$ Therefore, local vibration lowers muscle tension and the sensitivity to changes in muscle length, so we suggest that patients showed increased joint mobility after vibration therapy.

Based on the results of this study, it was found that it is

Table 2. The difference in shoulder horizontal adduction and internal rotation ROM between pre- and post-local vibration

$(\mathrm{N}=13)$

\begin{tabular}{cccc}
\hline \multirow{2}{*}{ ROM } & \multicolumn{2}{c}{ Mean $\pm \mathrm{SD}\left(^{\circ}\right)$} & \multirow{2}{*}{$p$} \\
\cline { 2 - 3 } & Pre-LV & Post-LV & \\
\hline HA & $17.7 \pm 6.8$ & $40.8 \pm 4.8$ & $<0.05^{*}$ \\
IR & $32.4 \pm 6.7$ & $59.3 \pm 5.3$ & $<0.05^{*}$ \\
\hline
\end{tabular}

ROM, range of motion; SD, standard deviation; HA, horizontal adduction; IR, internal rotation; LV, local vibration. effective to apply local vibrations to areas with shortening and stiffness of muscle or fascia tissue. In particular, when treating a region with limited mobility of a joint accompanied by shortening of soft tissue, it is believed that the effect will be high when applying a joint mobilization technique or muscle stretching after applying a local vibration first. This study has several limitations. First, the subjects who participated in this study consisted of only young ages, there are limitations to generalizing the results of the study. Second, this study only measured the immediate effect on $\mathrm{LV}$, it is considered to be somewhat insufficient to say that LV increases joint mobility. It is thought that research on long-term effects is also needed in the future. Third, only the effect of LV on joint mobility was dealt with, but future studies will need to study how it affects pain and movement.

\section{CONCLUSIONS}

Based on the results of this study, it was found that it is effective to apply LV to areas with shortening and stiffness of muscle or fascia tissue.

\section{Key Points}

Question What is the effect of local vibration on posterior shoulder tightness?

Findings The shoulder horizontal adduction and internal rotation ROM were significantly different between pre- and post-local vibration $(p<0.05)$.

Meaning Based on the results of this study, it was found that it is effective to apply local vibrations for improving posterior shoulder range of motion.

\section{Article information}

Conflict of Interest Disclosures: None.

Funding/Support: None.

Acknowledgment: None.

Ethic Approval: This study was approved by the Yonsei University, Mirae Campus, Institutional Review Board (approval number: 1041849-201701- BM-008-02).

\section{REFERENCES}

1. McClure P, Balaicuis J, Heiland D et al. A randomized controlled comparison of stretching procedures for posterior shoulder tightness. J Orthop and Sport Phys Ther. 2007;37(3):108-114.

2. Ludewig PM, Cook TM. Translations of the humerus in persons with shoulder impingement symptoms. J Orthop 
Sport Phys Ther. 2002;32(6):248-259.

3. Tyler TF, Roy T, Nicholas SJ, Gleim GW. Reliability and validity of a new method of measuring posterior shoulder tightness. J Orthop Sport Phys Ther. 1999;29(5):262-274.

4. Tyler TF, Nicholas SJ, Roy, T., Gleim, G.W. Quantification of posterior capsule tightness and motion loss in patients with shoulder impingement. Am J Sports Med. 2000;28(5):668-673.

5. Nelson, RT., Bandy, WD. Eccentric Training and static stretching improve hamstring flexibility of high school males. J Athl Train 2004;39(3):254-258.

6. Weppler $\mathrm{CH}$, Magnusson SP. Increasing muscle extensibility: a matter of increasing length or modifying sensation? Phys Ther. 2010;90(3):438-449.

7. Folpp H, Deall S, Harvey LA. et al. Can apparent increases in muscle extensibility with regular stretch be explained by changes in tolerance to stretch? Aust $J$ Physiother. 2006;52(1):45-50.

8. de Weijer VC, Gorniak GC, Shamus E. The effect of static stretch and warm-up exercise on hamstring length over the course of 24 hours. J Orthop Sport Phys Ther. 2003;33(12):727-733.

9. Pornratshanee W, Patria AH, Gregory S. Stretching: mechanisms and benefits for sport performance and injury prevention. Physical Therapy Reviews. 2004;9(4);189206.

10. Park KN, Kwon OY, Weon JH. Comparison of the effects of local cryotherapy and passive cross-body stretch on extensibility in subjects with posterior shoulder tightness. J Sports Sci Med. 2014;13(1):84-90.

11. Brodowicz GR, Welsh R, Wallis J. Comparison of stretching with ice, stretching with heat, or stretching alone on hamstring flexibility. $J$ Athl Train. 1996;31(4):324327.

12. Kim JY, Sung DJ, Lee JH. Therapeutic effectiveness of instrument-assisted soft tissue mobilization for soft tissue injury: mechanisms and practical application. $J$ Exerc Rehabil. 2017;13(1):12-22.

13. Zachary KP, Jason MD. The effects of acute and prolonged muscle vibration on the function of the muscle spindle's reflex arc. Somatosens Mot Res. 2015;32(4): 254-261.

14. Muharrem C, Ilhan K, Halil I, et al. Vibration parameters affecting vibration-induced reflex muscle activity. Somatosens Mot Res. 2017;34(1):47-51.

15. Mark H, Christopher C, Page S. Pacinian sgnals determine the direction and magnitude of the effect of vibration on pain. Perception. 2017;46(8):987-999. 